\title{
Vibro-impact in rolling contact
}

\author{
L.A. Sosnovskiy ${ }^{\mathrm{a}, *}$, S.S. Sherbakov ${ }^{\mathrm{b}}$ \\ ${ }^{a}$ Scientific and Production Group TRIBOFATIGUE Ltd., Apt. 42, Volotovskaya St. 4, 246050,Gomel, Belarus \\ ${ }^{\mathrm{b}}$ Belarusian State University, Apt. 42, Biruzova St. 11a, 220079, Minsk, Belarus
}

Accepted 12 April 2007

The peer review of this article was organized by the Guest Editor

\begin{abstract}
Irregular wavy residual damages (troppy phenomenon) occur in the contact area in rolling friction as the result of a nonstationary process of cyclic deformation. They initiate vibro-impact loading of an active system. The results of experimental study of these damages are given. Theoretical model satisfactory describing troppy phenomenon is developed.
\end{abstract}

(C) 2007 Elsevier Ltd. All rights reserved.

\section{Introduction}

It is shown in Refs. [1,2] that active systems of a roller/shaft type (Fig. 1) in bench tests for mechano-rolling fatigue [3] (Fig. 2) reproduce to a certain extent the conditions of operation of wheel/rail systems: both cyclic stresses under volume deformation (bending by load $Q$ ) and a field of contact stresses under the effect of contact load $F_{N}$ occur simultaneously in a working zone. Earlier (in 1996) such tests revealed a specific type of the limiting state: the appearance of residual wavy damages along the rolling track [4]. This phenomenon initially did not have any satisfactory explanation; no analytic description was provided. Below are the results of the new systematic experimental and theoretical analysis of the phenomenon considered.

\section{Experimental study}

A steel 25XTT (roller)/steel 45 (shaft) active system was tested for mechano-rolling fatigue. Steel 25XTT characteristics were the following: the fatigue limit $\sigma_{-1}=570 \mathrm{MPa}$, the rolling fatigue limit $p_{f}=3100 \mathrm{MPa}$. Steel 45 properties were the following: $\sigma_{-1}=260 \mathrm{MPa}$ and $p_{f}=1760 \mathrm{MPa}$. Thus, the characteristic feature of this active system is that the strength of the shaft's metal is significantly lower than that of the roller's; hence, the tests revealed residual deformations and damages only in the vicinity of the rolling track along the shaft, meanwhile the dimensions of the roller remained practically undistorted.

*Corresponding author. Tel./fax: + 375232774455

E-mail addresses: sosnovskiy@tribo-fatigue.com (L.A. Sosnovskiy), sersher@tut.by (S.S. Sherbakov). 


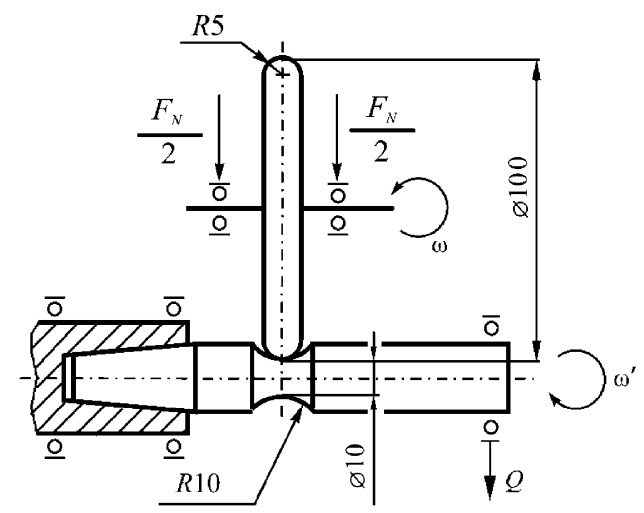

Fig. 1. Scheme of tests for mechano-rolling fatigue.

The amplitude of stresses $\sigma_{a}=225 \mathrm{MPa}<\sigma_{-1}=260 \mathrm{MPa}$ corresponds to the bending load $Q=225$, $N=$ const. The contact load changed step by step according to the program indicated in Fig. 2 The rolling fatigue limit $p_{f}=1760 \mathrm{MPa}$ was exceeded at step III of loading.

The motion of the roller on the shaft became non-stationary during the transition from step VII to step VIII of contact loading, i.e. after 700,000 cycles of the test (see arrow 1 in Fig. 2).

The loss of motion stability took place at step IX (see arrow 2 in Fig. 2). It led to initiation of vibro-impact process. The tests were discontinued at step IX at $N_{\Sigma}=976,100$ cycles of loading because of inadmissible vibration and noise. The shaft's axial $a_{x}$ and radial $h_{z}$ deformations after the tests are shown in Figs. 3 and 4.

The main features of the shaft's damage are the following (Fig. 5). Several peculiar "frozen" waves of surface plastic deformation appear along the rolling track. They represent a combination of irregular craters of the half-barrel shape. None of the craters repeat and each have its unique dimensions in all three radial, axial, and peripheral directions. The magnitude of the relative plastic deformation (in the test conditions) reaches $14 \%$ in the radial and $32 \%$ in the axial directions.

Thus, in these conditions of tests, the residual surface wavy damages can be treated as the result of a nonstationary process of cyclic elastoplastic deformation in the zone of contact interaction between two elements of the system. This phenomenon has acquired the name "troppy" [5], and this non-stationary process is accompanied by three-dimensional distortions of the dimensions of the rolling track on both the roller and the shaft and different local resistance of the material in different "points" along the rolling track. Wavy damages along the rolling track are shown in Fig. 6a. The strength of the material rated, for example, as hardness, is significantly different in these points before and after the tests. The change of hardness along the curve $L$ (Fig. 6a) that belongs to the perimeter of the shaft's cross-section is shown in Fig. $6 \mathrm{~b}$.

Note that the phenomenon under study is inversed in the following sense: if the roller's material strength is significantly less than that of the shaft's, then the residual wavy damages are formed on the roller [6], whereas the dimensions of the shaft's rolling path remain almost undistorted. In the case that the roller and the shaft are made of one type of steel and have identical surface properties, residual wavy damages in certain test conditions are formed on the working surfaces of both elements simultaneously.

\section{Theoretical description}

From the theoretical viewpoint, the described damage is the result of stability loss of the roller motion on the shaft. It is characterized by self-excitation of oscillations in the system and accompanied by cyclic impact elastoplastic deformation of bodies in the contact region.

\subsection{Basic concepts}

The following general assumptions based on the experimental results described above are considered in the theoretical solution 

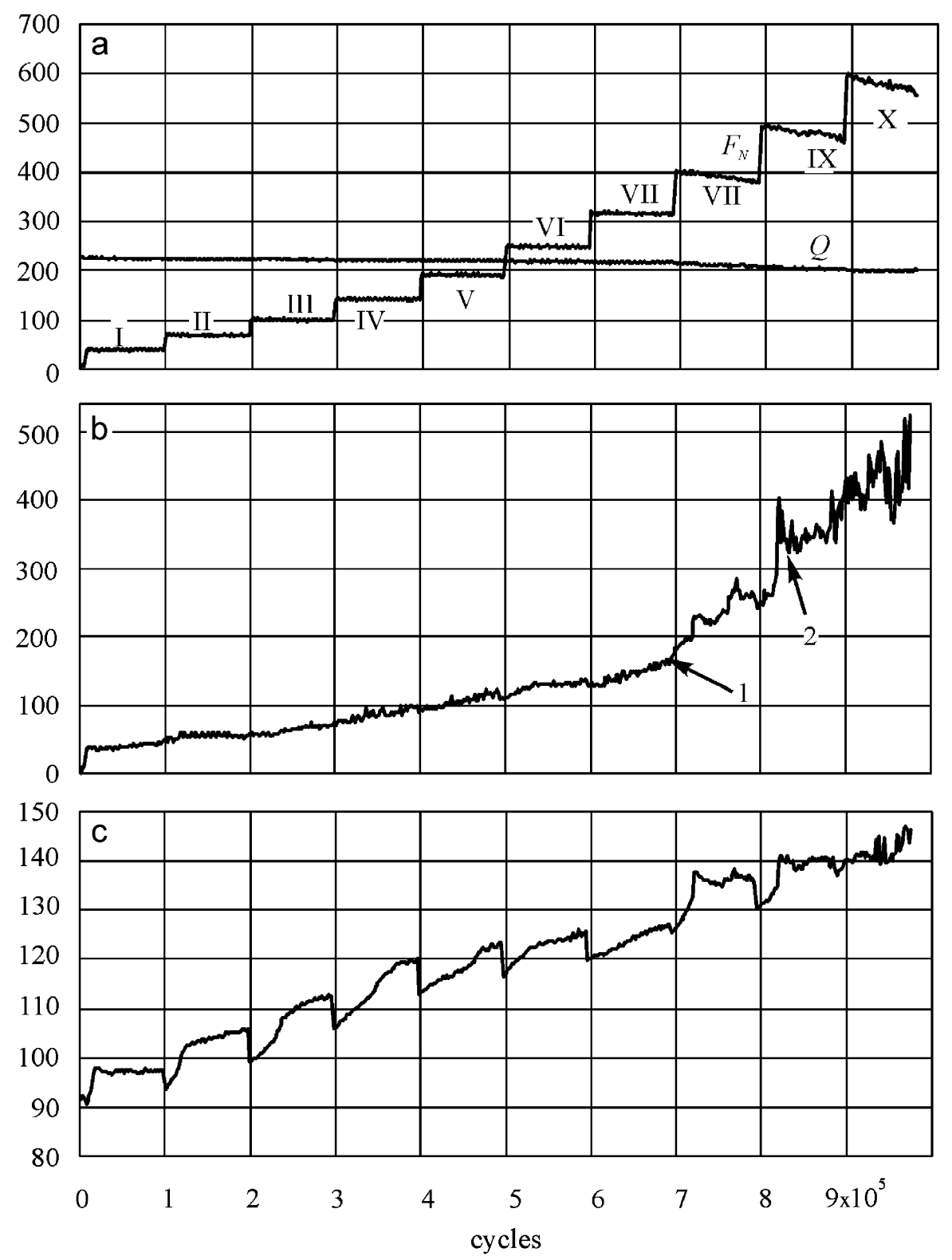

Fig. 2. Protocol of tests of roller (steel 25XTT)/shaft (steel 45) system for mechano-rolling fatigue: (a) loading program $F_{\mathrm{N}}, Q(N),(b)$ axes displacement $\delta(\mu \mathrm{m})$, and (c) noise $(\mathrm{dB})$.

1. Load $F_{N}$ causes both local contact deformations and deformation of the shaft as a whole (Fig. 7).

2. Stresses caused by contact and non-contact loads in the roller/shaft active system act simultaneously and in the same area in the neighborhood of contact area of the roller and the shaft.

3. The excitation of roller oscillations during motion may occur in planes $x z$ and $x y$ and along axis $z$ (Fig. 7a).

4. Main contact parameters: semi-axes $a$ and $b$ of the contact ellipse, maximum pressure magnitude $p_{0}$ in the center of contact area, displacement of axes $\delta$ change as the result of motion stability loss.

5. Motion stability loss in time may also be initiated by the change of local mechanical properties along the rolling path.

6. Generally both disturbing effect of different loading factors and change in time of geometrical dimensions in the contact area are taken into account for the description of motion stability loss. These geometry changes may result from residual deformations. 


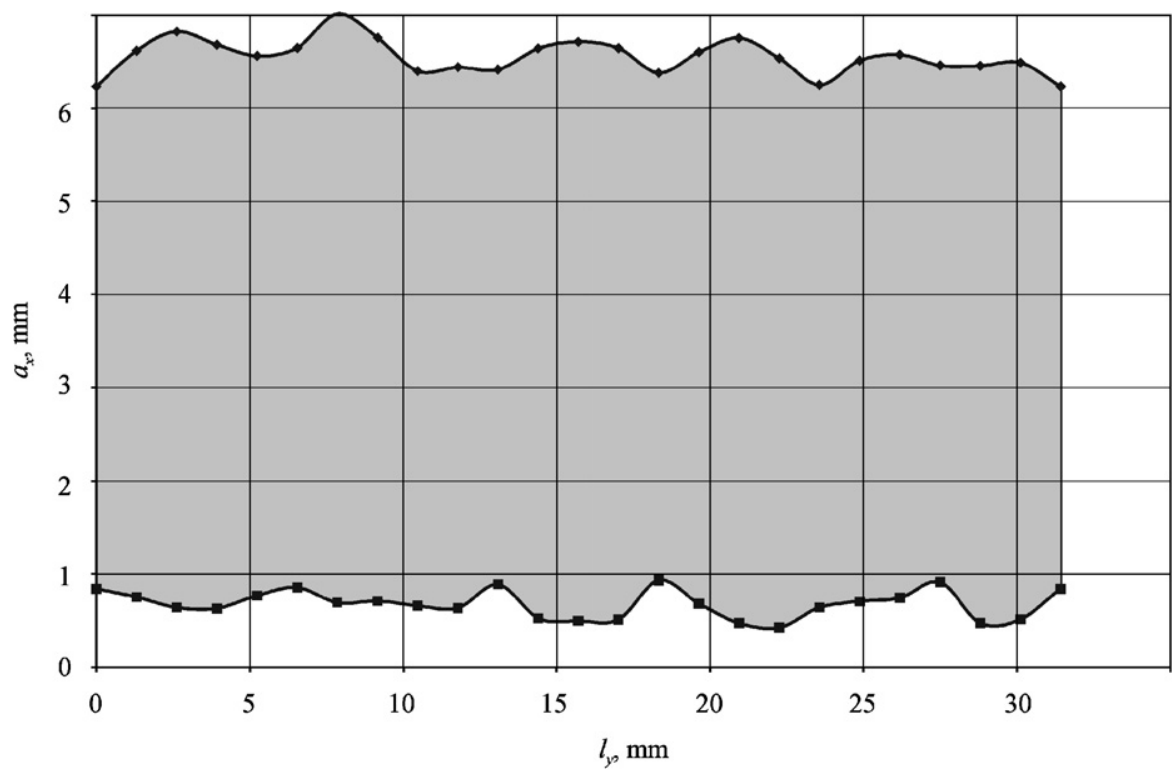

Fig. 3. Axial residual wavy deformation after tests for mechano-rolling fatigue $\left(N_{\Sigma}=976,100\right.$ cycles $)$ along the rolling track of the length $L=2 \pi R_{21}\left(R_{21}=5 \mathrm{~mm}\right)$.

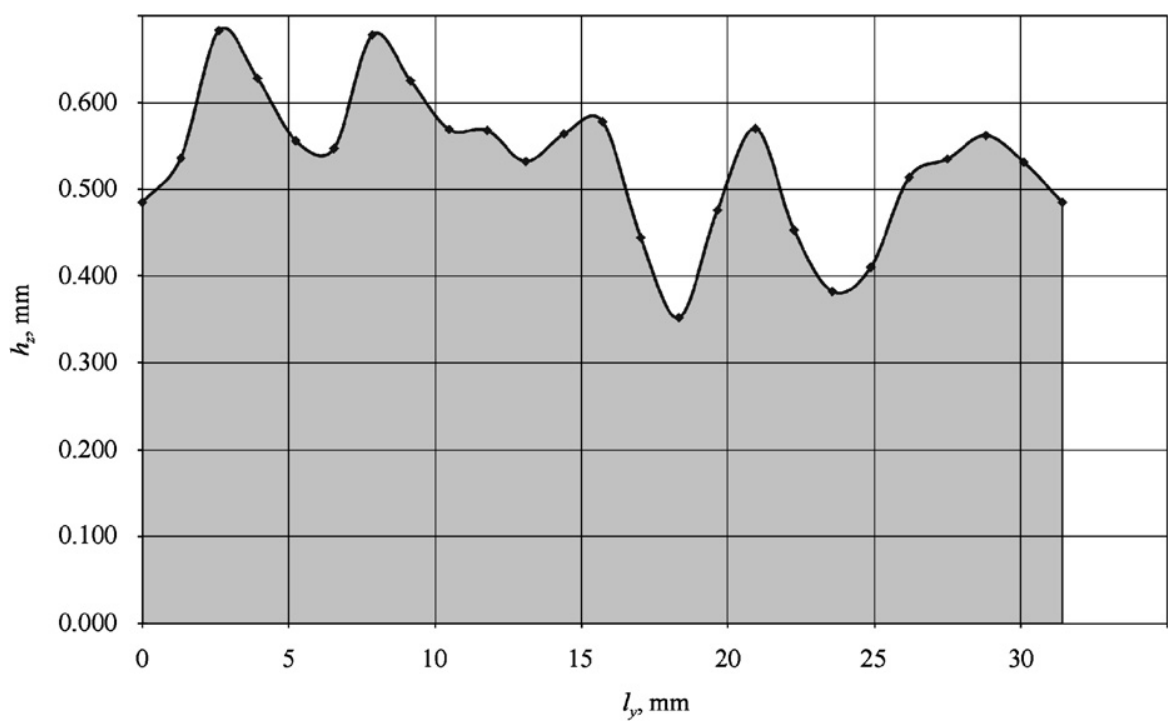

Fig. 4. Radial residual deformation after tests for mechano-rolling fatigue $\left(N_{\Sigma}=976,100\right.$ cycles $)$ along the rolling track $L$.

Thus, the concepts formulated for theoretical study are adequate to the test results and are the extension of experimental research.

\subsection{Statement of the problem}

The following conditions are taken into consideration when building the equations of oscillations for the roller in the planes $x z$ and $x y$ (Fig. 7):

1. The roller is elastically fixed on its axis of rotation in such a manner that restoring elastic moments $k_{\psi} \psi$ and $k_{\theta} \theta$ are proportional to the angles of deviation $\psi$ and $\theta$ (here $k_{\psi}$ and $k_{\theta}$ are coefficients of proportionality [8]). 

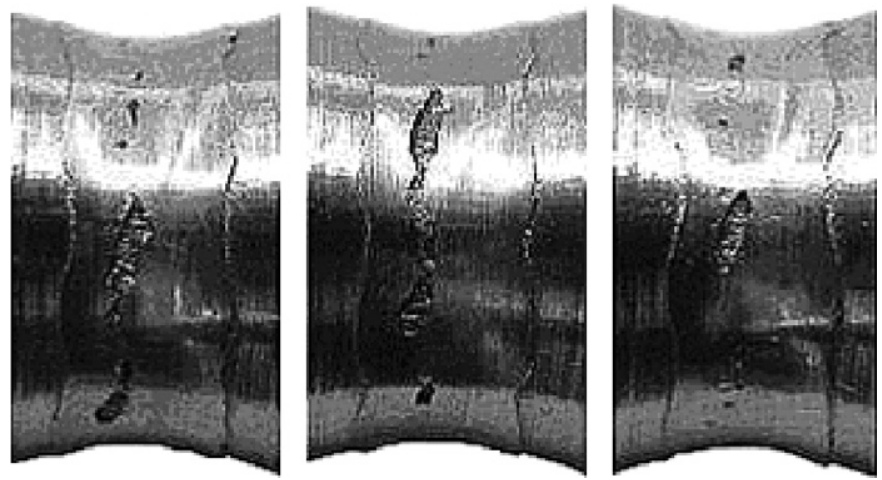

Fig. 5. Pattern of shaft damage after the tests for mechano-rolling fatigue.

a

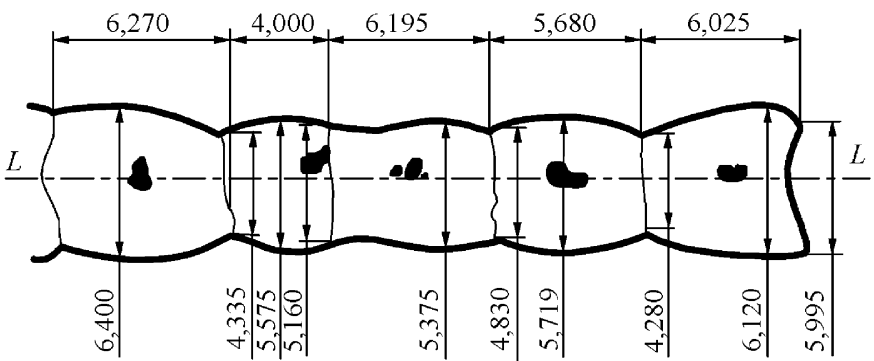

b

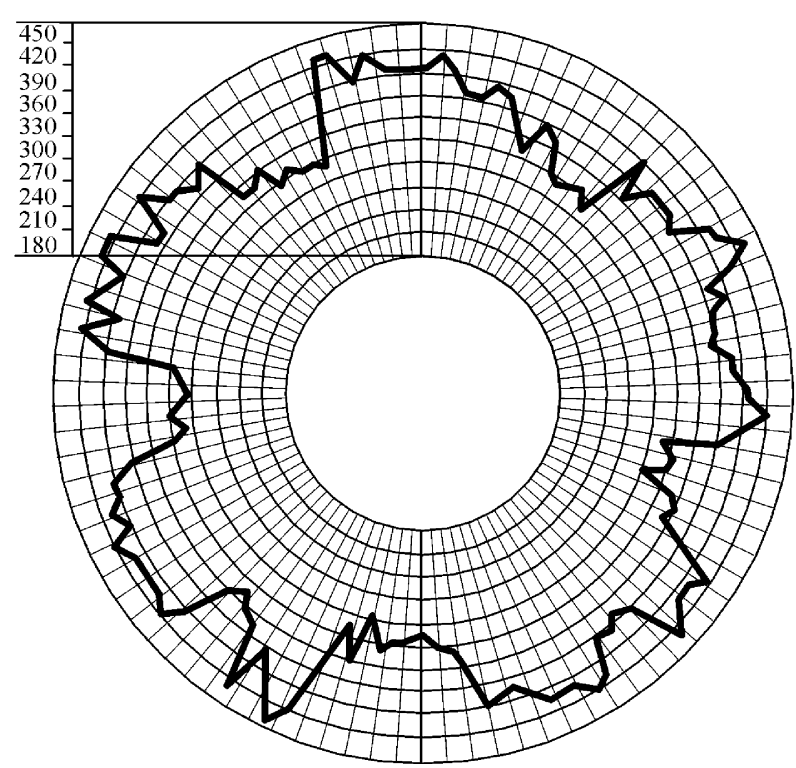

Fig. 6. Surface residual wavy damages (pittings are shown black) (a) and distribution of microhardness along the rolling track $L$ on the shaft (b).

2. The roller is in the state of equilibrium in the condition of unexcited movement $\psi=\theta=0$.

3. The roller's and the shaft's angular velocities $\omega$ and $\omega^{\prime}$ are considered preset. Their magnitudes during the tests may be chosen to minimize sliding between the roller and the shaft.

4. When deviated from the state of equilibrium the roller oscillates around vertical axis $z$ and axis $y$ parallel to the direction of motion. 
a

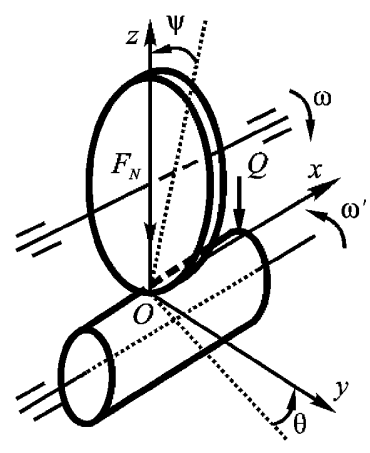

b

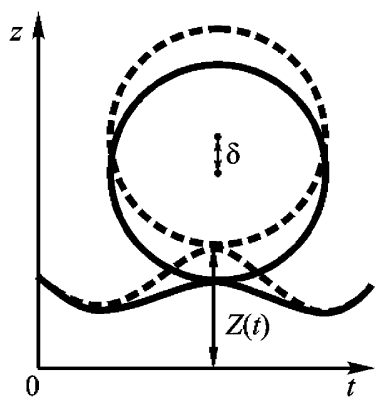

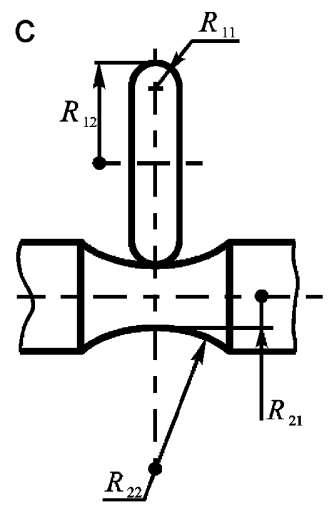

Fig. 7. Calculation schemes (a) and (b) and a designation of radii of curvature (c).

Only some works are known in which similar particular problems have been considered. Ref. [6] gives the solution for the problem of static concentrated force applied to a beam lying on two supports. In this case, both local (contact) deformation in the neighborhood of the point of force application and deformation of the beam as a whole occur. In Ref. [7], Timoshenko's solution for collision of two elastic bodies (spherical indenter and a beam) is considered. No wavy damages occur in these two cases.

Both the law of motion of the roller on the shaft in three-dimensional space according to assumption 3 and a three-dimensional state of stress caused by contact and non-contact loading according to assumptions 1, 2, and 4 should be studied for considered active system. Consider the following approach to determine loading of an active system when force $F_{N}$ causes both local contact deformation and volume deformation of the shaft.

Assume that in the contact region $F_{N}$ is divided into contact $F_{c}$ and bending components $F_{b}$ under the following condition:

$$
F_{N}=F_{c}+F_{b}
$$

Consider the condition of energy balance of a deformable rigid body as the equality of strain energy and work performed by surface forces at corresponding displacements in the absence of volumetrically distributed loads in the body:

$$
\iiint_{V} \mathbf{T}: \mathbf{E} \mathrm{d} V+\iint_{S} \mathbf{F u} \mathrm{d} S=0
$$

where $\mathbf{T}: \mathbf{E}$ is a biscalar product of stresses tensor $\mathbf{T}$ and deformations tensor $\mathbf{E}, \mathbf{F}$ the surface forces, and $\mathbf{u}$ the surface displacements.

Load $F_{N}$ is a surface force and such are $F_{c}$ and $F_{b}$. Due to the particularities of contact interaction, $F_{c}$ actually causes distributions of contact pressure $p\left(F_{c}, S\right)$ and displacement $\mathbf{u}\left(F_{c}, S\right)$. However, in order to simplify calculations we will consider forces $F_{c}$ and $F_{b}$ applied at a point causing corresponding displacements $u_{c}$ and $u_{b}$.

To determine the magnitudes of $F_{c}$ and $F_{b}$ in terms of Eq. (1) and condition of energy balance (2), the general system of equations is written in the following way:

$$
\begin{aligned}
& \iiint_{V} \mathbf{T}: \mathbf{E} \mathrm{d} V+F_{N} u_{N}=0, \\
& F_{c}+F_{b}=F_{N} .
\end{aligned}
$$

Examine the explicit form of the condition of energy balance in Eq. (3):

$$
\iiint_{V} \mathbf{T}: \mathbf{E} \mathrm{d} V+F_{N} u_{N}=\iiint_{V}\left(\mathbf{T}^{(c)}+\mathbf{T}^{(b)}\right):\left(\mathbf{E}^{(c)}+\mathbf{E}^{(b)}\right) \mathrm{d} V+\left(F_{c}+F_{b}\right)\left(u_{c}+u_{b}\right)=0
$$


or

$$
\iiint_{V} \mathbf{T} \cdot \mathbf{E} \mathrm{d} V+F_{N} u_{N}=\iiint_{V}\left[\sum_{i, j}\left(\sigma_{i j}^{(c)}+\sigma_{i j}^{(b)}\right)\left(\varepsilon_{i j}^{(c)}+\varepsilon_{i j}^{(b)}\right)\right] \mathrm{d} V+\left(F_{c}+F_{b}\right)\left(u_{c}+u_{b}\right)=0 .
$$

In case linear dependence between stresses and strains is taken into account

$$
u_{c}=k_{c} F_{c}^{3 / 2}, \quad u_{b}=k_{b} F_{b},
$$

we obtain

$$
\begin{aligned}
& E \iiint_{V}\left\{\sum_{i, j}\left[\left(\sigma_{i j}^{(c)}\right)^{2}+2 \sigma_{i j}^{(c)} \sigma_{i j}^{(b)}+\left(\sigma_{i j}^{(b)}\right)^{2}\right]\right\} \mathrm{d} V+k_{c} F_{c}^{5 / 2} \\
& +\left(k_{b}+k_{c} \sqrt{F_{c}}\right) F_{c} F_{b}+k_{b} F_{b}^{2}=0 .
\end{aligned}
$$

Considering that $\sigma_{i j}^{(c)}=\sigma_{i j}\left(F_{c}\right), \sigma_{i j}^{(b)}=\sigma_{i j}\left(F_{b}\right)$ and using Eq. (1) the following equation can be written

$$
\begin{aligned}
& E \iiint_{V}\left\{\sum_{i, j}\left[\sigma_{i j}^{2}\left(F_{c}\right)+2 \sigma_{i j}\left(F_{c}\right) \sigma_{i j}\left(F_{N}-F_{c}\right)+\sigma_{i j}^{2}\left(F_{N}-F_{c}\right)\right]\right\} \mathrm{d} V \\
& \quad+k_{c} F_{c}^{5 / 2}+\left(k_{b}+k_{c} \sqrt{F_{c}}\right) F_{c}\left(F_{N}-F_{c}\right)+k_{b}\left(F_{N}-F_{c}\right)^{2}=0 .
\end{aligned}
$$

The magnitude of contact component $F_{c}$ can be determined using Eq. (7). Then using Eq. (1) $F_{b}$ can be obtained.

\subsection{Motion of active system}

Moments of inertia of the roller about axes $y$ and $z$ and centrifugal moment are designated by $I_{y}, I_{z}$, and $I_{y z}$, respectively. The moment of inertia of the roller about its axis of rotation $x$ is designated by $i$. Then with regard to Refs. [8,9] the system of roller's motion equation for small oscillations can be written in the following form:

$$
\begin{aligned}
& I_{y} \ddot{\psi}+I_{y z} \ddot{\theta}+\mathrm{i} \omega \dot{\theta}=-k_{\psi} \psi+M_{\psi}, \\
& I_{y z} \ddot{\psi}+I_{z} \ddot{\theta}-\mathrm{i} \omega \dot{\psi}=-k_{\theta} \theta+M_{\theta}, \\
& \ddot{z}+\kappa^{2} z=\kappa^{2} Z(t) .
\end{aligned}
$$

Here $M_{\psi}, M_{\theta}$ are moments about the axes $y$ and $z$ applied to the roller in the contact area, $\mathrm{i} \omega \dot{\theta}, \mathrm{i} \omega \dot{\psi}$ are gyroscopic moments about the axes $y$ and $z, Z(t)$ the law of change of kinetic disturbance, and $\kappa$ a coefficient.

\subsection{Parameters of contact region}

The contact region parameters can be determined by solving Hertz contact problem for a couple of bodies that come into elliptic contact being pressed together by force $F_{c}[10]$.

Expressions for semi-axes of the contact ellipse $a$ and $b$, the greatest pressure $p_{0}$ in the center of contact area, and axes displacement $\delta$ for the case of static contact can be found in Ref. [10]. In our case, we will consider contact area parameters as the time-dependent functions:

$$
a=a(e(t)), \quad b=b(e(t)), \quad \delta=\delta(e(t)), \quad p_{0}=p_{0}(e(t)),
$$

where we will calculate the eccentricity $e$ of contact ellipse according to the formula [11]

$$
\begin{aligned}
e^{2}(\Omega)= & e\left(\Omega\left(R_{11}(t), R_{12}(t), R_{21}(t), R_{22}(t), \theta(t)\right)\right) \\
= & 2,667 \Omega-3,577 \Omega^{2}+4,244 \Omega^{3}-5,871 \Omega^{4}+10,312 \Omega^{5}-18,202 \Omega^{6} \\
& +24,577 \Omega^{7}-21,858 \Omega^{8}+11,213 \Omega^{9}-2,506 \Omega^{10}
\end{aligned}
$$


where

$$
\Omega=\frac{1}{\sum k} \sqrt{\left(k_{11}-k_{12}\right)^{2}+\left(k_{21}-k_{22}\right)^{2}+2\left(k_{11}-k_{12}\right)\left(k_{21}-k_{22}\right) \cos 2 \alpha},
$$

$k_{i j}(i j=1,2)$ are the main curvatures of contacting bodies, $\alpha=\pi / 2-\theta$ is the angle between $k_{11}$ and $k_{21}$.

\subsection{Loading factors}

In the accepted statement of the problem loading factors are the moments of forces transmitted to the roller on the contact surface.

The integral moment caused by distribution of normal traction $p(x, y)$ over the area of contact $S$ with an $\operatorname{arm} h(x, y)$ is

$$
M(x, y)=\iint_{S} h(x, y) p(x, y) \mathrm{d} x \mathrm{~d} y .
$$

In order to simplify integration, it is expedient to use the following connection between the coordinate system connected rigidly with the ellipse of contact and global coordinate system as the ellipse of contact turns by angles $\theta$ and $\psi$ (Fig. 8):

$$
x=x^{\prime} \cos \theta+y^{\prime} \sin \theta+R \sin \psi, \quad x=-x^{\prime} \sin \theta+y^{\prime} \cos \theta, \quad z=z^{\prime},
$$

where $R=R_{12}$ is a greater radius of the roller; $x, y, z$ represent a global coordinate system $x^{\prime}=\operatorname{ar} \cos \varphi$, $y^{\prime}=b r \sin \varphi$, and $z^{\prime}$ represent a local coordinate system connected rigidly with the ellipse of contact.

With regard to Eq. (12) the expression (11) becomes

$$
M_{\psi}^{p}\left(x^{\prime}, y^{\prime}\right)=\iint_{S^{\prime}} h_{x}\left(x\left(x^{\prime}, y^{\prime}\right)\right) p\left(x^{\prime}, y^{\prime}\right) \mathrm{d} x^{\prime} \mathrm{d} y^{\prime} .
$$

Differential of the moment about the $y$-axis in local coordinate system is

$$
\mathrm{d} M_{\psi}^{p}=(R \sin \psi+a r \cos \varphi \cos \theta+b r \sin \varphi \sin \theta) p_{0} \sqrt{1-r^{2}} a b r \mathrm{~d} r \mathrm{~d} \varphi .
$$

Hence, the moment is

$$
M_{\psi}^{p}=\int_{0}^{2 \pi} \int_{0}^{1}(R \sin \psi+a r \cos \varphi \cos \theta+b r \sin \varphi \sin \theta) p_{0} \sqrt{1-r^{2}} a b r \mathrm{~d} r \mathrm{~d} \varphi .
$$

Integral (15) is easy to find:

$$
M_{\psi}^{p}=\frac{2 \pi}{3} p_{0} a b R \sin \psi=F_{N} R \sin \psi .
$$

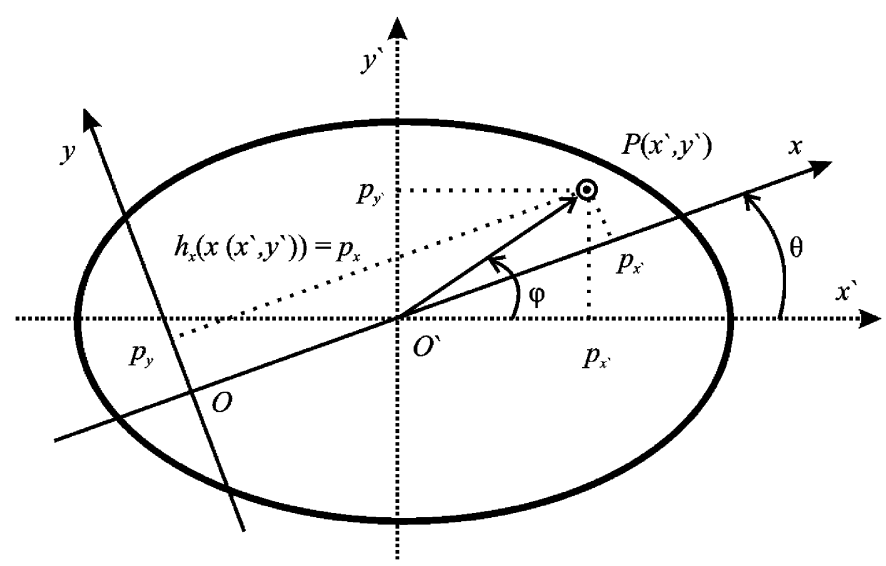

Fig. 8. To determination of moment $M_{\psi}{ }^{p}$ 
From here it is evident that since the integral over $\varphi \in[0 \ldots 2 \pi]$ of functions sin and cos is equal to 0 , the obtained expression does not depend on $\theta$. It is apparent from the fact that the total moment about the $y$-axis remains constant when the roller rotates about the $z$-axis.

The moments $M_{\psi}^{\sigma}, M_{\theta}^{\sigma}$ caused by the action of bending load $Q$ and the moment $M_{\theta}^{f p}$ caused by force of friction are calculated similarly:

$$
\begin{aligned}
M_{\psi}^{\sigma} & =-\frac{f_{0} Q \pi a b h}{2 J_{y}} R \cos \psi(A-1+R \cos \psi), \\
M_{\theta}^{\sigma} & =\frac{f_{0} Q \pi a b h}{4 J_{y}}\left(a^{2}-b^{2}\right) \sin 2 \theta, \\
M_{\theta}^{f p} & = \pm f_{r} F_{N} R \sin \psi .
\end{aligned}
$$

where $J_{y}=\pi r^{4} / 4$ is the moment of inertia of the shaft of a circular cross-section, $h=R_{21}-\delta, A$ the distance from the point of cantilever fixing of the shaft to the center of the initial contact, and $f_{r}, f_{0}$ are the coefficients of rolling and sliding friction, respectively.

\section{Troppy model}

Basing on Eqs. (8), (16), and (17) the general system of differential equations of motion can be written in the following way:

$$
\begin{aligned}
& I_{y} \ddot{\psi}+I_{y z} \ddot{\theta}+\mathrm{i} \omega \dot{\theta}=-k_{\psi} \psi+M_{\psi}^{p}+M_{\psi}^{\sigma}, \\
& I_{y z} \ddot{\psi}+I_{z} \ddot{\theta}-\mathrm{i} \omega \dot{\psi}=-k_{\theta} \theta+M_{\theta}^{f p}+M_{\theta}^{\sigma}, \\
& \ddot{z}+\kappa^{2} z=\kappa^{2} Z(t) .
\end{aligned}
$$

The first two equations of the system (18) are Lagrange equations of the second kind, with the second members corresponding to our statement of the problem. Keldysh equations for shimmy [8] were used for their formulation.

The moments of the forces applied to the roller on the surface of contact

$$
\begin{aligned}
M_{\psi}^{p} & =M_{\psi}^{p}\left(F_{N}, \psi(t)\right), \\
M_{\psi}^{\sigma} & =M_{\psi}^{\sigma}(Q(t), a(t), b(t), \delta(t), \psi(t)), \\
M_{\theta}^{\sigma} & =M_{\theta}^{\sigma}(Q(t), a(t), b(t), \delta(t), \theta(t)), \\
M_{\theta}^{f p} & =M_{\theta}^{f p}\left(F_{N}, f_{r}, \psi(t)\right) .
\end{aligned}
$$

The system (18) is built in terms of analytical dependence (10) of contact ellipse eccentricity $e$ on the radii $R$ of curvature of bodies in the point of initial contact. This dependence defines the change of contact parameters in time.

In that specific case, when parameters $a, b, p_{0}$, and $\delta$ do not depend on time, Eq. (18) with regard to Eqs. (16) and (17) are

$$
\begin{aligned}
& I_{y} \ddot{\psi}+I_{y z} \ddot{\theta}+\mathrm{i} \omega \dot{\theta}=-k_{\psi} \psi+F_{N} R \sin \psi-\frac{f_{0} Q \pi a b h}{2 J_{y}} R \cos \psi(A-1+R \cos \psi), \\
& I_{y z} \ddot{\psi}+I_{z} \ddot{\theta}-\mathrm{i} \omega \dot{\psi}=-k_{\theta} \theta \pm f_{r} F_{N} R \sin \psi+\frac{f_{0} Q \pi a b h}{4 J_{y}}\left(a^{2}-b^{2}\right) \sin 2 \theta, \\
& \ddot{z}+\kappa^{2} z=\kappa^{2}\left[\frac{1}{2} a_{0}+\sum_{s=1}^{\infty}\left(a_{s} \cos s p t+b_{s} \sin s p t\right)\right],
\end{aligned}
$$

where $\frac{1}{2} a_{0}+\sum_{s=1}^{\infty}\left(a_{s} \cos s p t+b_{s} \sin s p t\right)$ is trigonometrical series by which kinetic disturbance causing oscillations along the $z$-axis can be generally represented.

The equations (20) take into account the roller's ability to oscillate in three planes, characteristic features of geometry of interacting elements at the contact interface, heterogeneity of surface properties of 
material in local zones along the rolling track, and disturbing action of external (contact and non-contact) loads.

\subsection{State of stress}

The state of stress in any point of the system during the motion of the roller along the shaft generally depends on time $t$, velocity of impact $v$ and height of asperities $h$ of wavy damage of the shaft and the roller. The state of stress is determined by the general expression

$$
\sigma_{i j}(t, v, h)=\sigma_{i j}^{(n)}(t, v, h)+\sigma_{i j}^{(\tau)}(t, v, h)+\sigma_{i j}^{(b)}(t, v, h), \quad i, j=x, y, z,
$$

where $\sigma_{i j}^{(n)}$ are the stresses caused by normal contact load, $\sigma_{i j}^{(\tau)}$ are the stresses caused by tangential contact load, $\sigma_{i j}^{\left(b^{h}\right)}$ are the stresses produced by non-contact loads.

\subsection{Dangerous volumes}

The analysis of the most general case of interaction of the elements of an active system and its damage is based on a statistical model of a deformable rigid body with dangerous volume [1].

The knowledge of (a) function of distribution of fatigue limits of a corresponding element of an active system and (b) the state of stress in zones of contact interaction of bodies is necessary for determining the shapes and magnitudes of dangerous volumes.

Dangerous volumes are the regions in which the stresses exceed the minimal values $\sigma_{-1 \text { min }}^{(n)}, \sigma_{-1 \text { min }}^{(\tau)}$ of fatigue limits for normal and tangential stresses, respectively.

Magnitudes of component dangerous volumes are specified according to the following formula after the calculation of the state of stress:

$$
V_{i j}=\iiint_{\sigma_{i j}(\xi, \eta, z)-\sigma_{-1 \min }^{(k)}>0} \mathrm{~d} \xi \mathrm{d} \eta \mathrm{d} z, \quad i, j=x, y, z,
$$

where

$$
\sigma_{-1 \min }^{(k)}= \begin{cases}\sigma_{-1 \min }^{(n)}, & \text { if } i=j \\ \sigma_{-1 \min }^{(\tau)}, & \text { if } i \neq j\end{cases}
$$

The magnitude of tensor dangerous volume is determined according to the expression:

$$
V_{T}=\bigcup_{i, j} V_{i j}
$$

Corresponding measures of damage are

$$
\omega_{i j}=V_{i j} / V_{k}, \quad \omega_{T}=V_{T} / V_{k},
$$

where $V_{k}$ is the working volume.

\section{Dynamic contact}

The solutions obtained allow the analysis of the dynamic contact to be made provided that the change of contact parameters in time is established.

If for example we change $R_{11}, R_{21}$, and $F_{N}$ in time as it is shown in Fig. 9, then contact parameters change according to Eq. (9), is as shown in Fig. 10. Here $X_{a b}$ and $Y_{a b}$ are the magnitudes of semi-axes of the contact ellipse along the axes $x$ and $y$, respectively.

The dependencies shown in Fig. 9 were chosen in such a way that the influence of $R_{i j}(t) i, j=1,2$ and $F_{N}(t)$ on the change of all contact parameters (Fig. 10) can be tracked. The exact form of dependency $R_{21}(t)$ was chosen in order to simulate oscillations of contact parameters. 


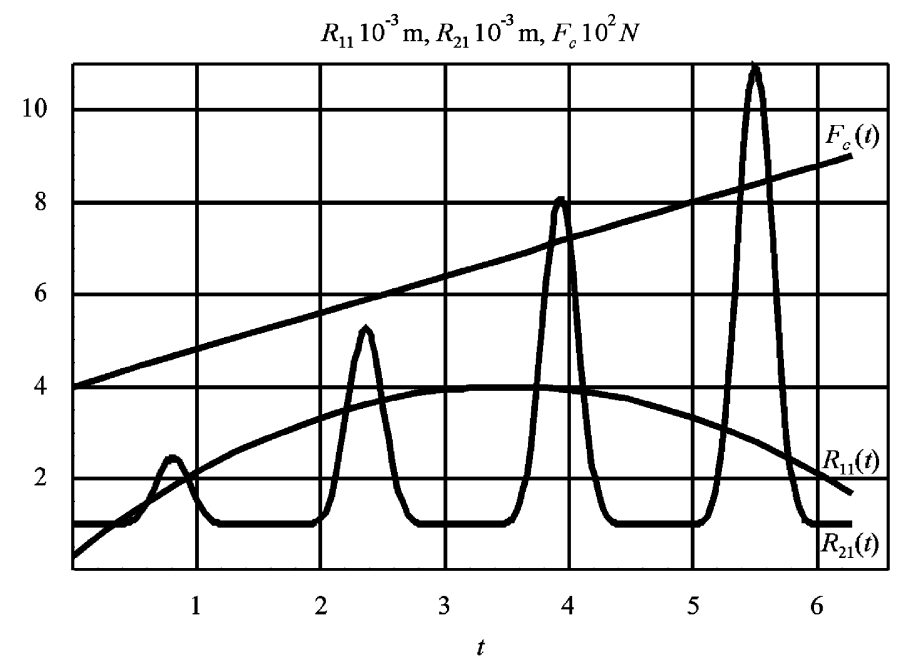

Fig. 9. Law of change of $R_{11}(t) 10^{-3} \mathrm{~m}, R_{21}(t) 10^{-3} \mathrm{~m}$, and $F_{N}(t) 10^{2} \mathrm{~N}$.

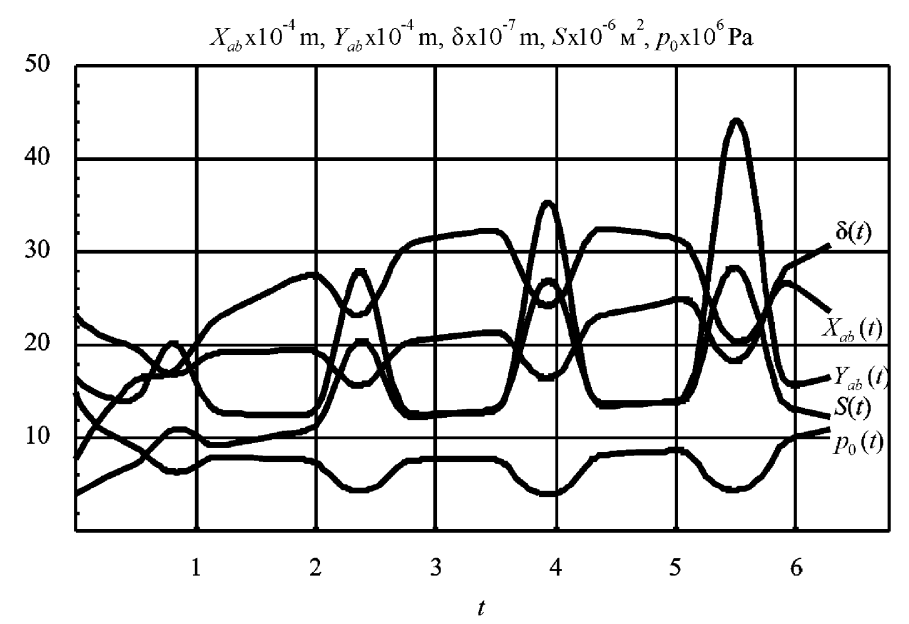

Fig. 10. Change in time of $X_{a b}(t) 10^{-4} \mathrm{~m}, Y_{a b}(t) 10^{-4} \mathrm{~m}, S(t) 10^{-6} \mathrm{~m}^{2}, p_{0}(t) 10^{6} \mathrm{~Pa}$, and $\delta(t) 10^{-7} \mathrm{~m}$.

The distributions of stresses $\sigma_{z z}^{(n)}$ and $\sigma_{x z}^{(n)}$ (four left figures) and dangerous volumes (two right figures) at the discrete moments of time in planes $z=0.3 a$ (top figures) and $y=0$ (bottom figures) (where $a$ is the magnitude of the greater semi-axis of the contact ellipse at the given moment of time) are shown in Figs. 11-14. Each group of four figures gives the exhaustive analysis of the state of stress at the given moment of time.

Figs. 11-14 show that stresses $\sigma_{z z}^{(n)}$ are greater in the vicinity of contact region so that condition $\max _{(x, y, z)}\left(\sigma_{z z}^{(n)}\right)=\sigma_{z z}^{(n)}(0,0,0)=p_{0}$ is satisfied. For example, if $t=0$ then $p_{0}=-1.4 \times 10^{8} \mathrm{~Pa}$. Maximum and minimum of tangential stresses $\sigma_{x z}^{(n)}$ are located at the depth of $0.27 a$ under the surface. Absolute extreme values of tangential stresses are $0.3 p_{0}$. For example, if $t=2$ then $\max _{(x, y, z)}\left(\left|\sigma_{x z}^{(n)}\right|\right)=-2 \times 10^{7} \mathrm{~Pa}$.

The analysis of Figs. 11-14 and Figs. 9 and 10 shows that the developed model makes it possible to describe the change of the shape and magnitude of stresses distribution as the result of time change of the applied load and bodies geometry in the contact area (Fig. 10). More detailed analysis of these results is given in Ref. [11].

Occurrence of vibro-impact depends first of all on the intensity and frequency of change $\delta(t)$ and $p_{0}(t)$. Rapid changes of the given parameters generate "bursts" of stresses magnitudes.

In the conditions of calculation, the planes to which radii $R_{11}$ and $R_{21}$ belong to are parallel to axes $y$ and $x$, respectively. Consider Figs. 11 and 13 and the corresponding points $t=0$ and 4 in Figs. 9 and 10. It is evident 

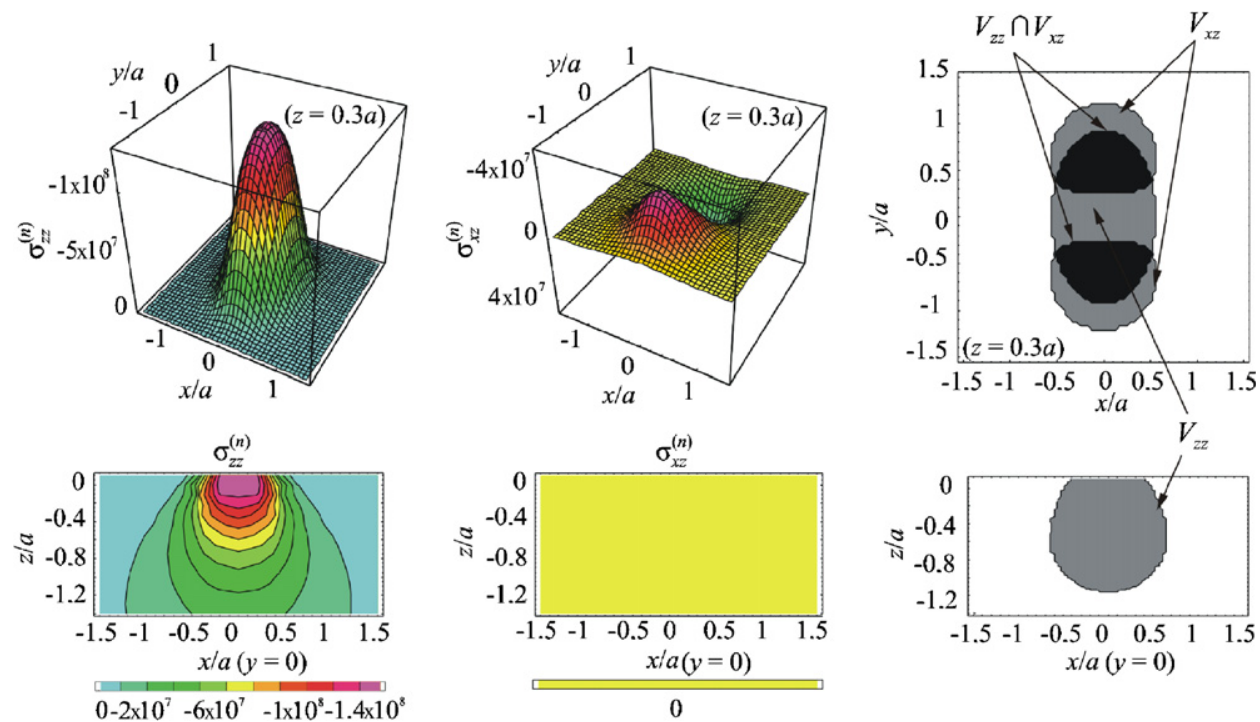

Fig. 11. Distribution of stresses $\sigma_{z z}$ and $\sigma_{x z}$ and respective dangerous volumes in planes $z=0.3$ a (top row) and $y=0$ (bottom row) at initial moment of time $(t=0)$.
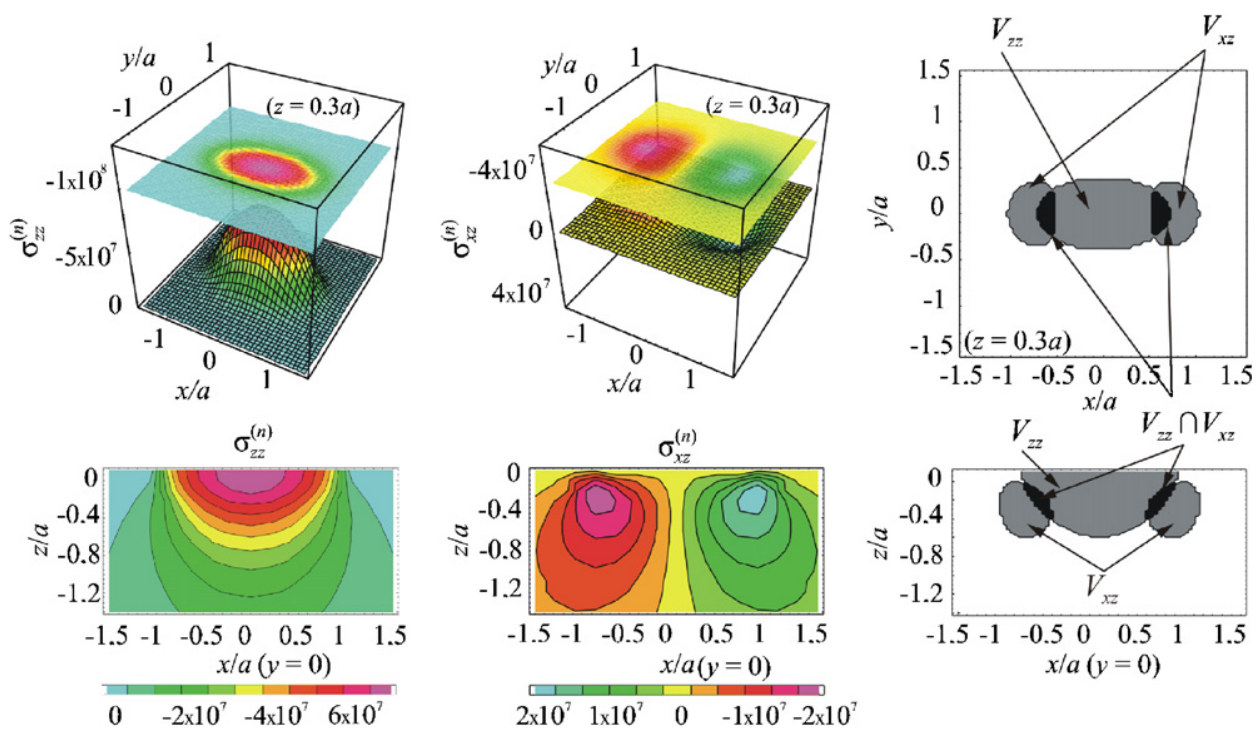

Fig. 12. Distribution of stresses $\sigma_{z z}$ and $\sigma_{x z}$ and respective dangerous volumes in planes $z=0.3$ a (top row) and $y=0$ (bottom row) at moment of time $t=2$.

that if $R_{11}>R_{21}$ (Fig. 9) the greater semi-axis of the contact ellipse is oriented along $y$-axis (the distribution of stresses in Fig. 11 as well) i.e. $Y_{a b}>X_{a b}$. As this takes place, the magnitude of stresses at a point $t=0$ is greater than at a point $t=4$ because $S(1)<S(4)$ and $p_{0}(1)>p_{0}(4)$. The relations inverse to the described ones take place at the moments of time $t=2$ and 6 .

Dangerous volumes (right part of Figs. 11-14) formed by stresses $\sigma_{z z}^{(n)}$ and $\sigma_{x z}^{(n)}$ for the respective limiting normal $\sigma_{-1 \lim }^{n}=4 \times 10^{7} \mathrm{~Pa}$ and tangent $\sigma_{-1 \mathrm{lim}}^{7}=10^{7} \mathrm{~Pa}$ stresses have corresponding magnitude and orientation. The darker parts of dangerous volumes correspond to the areas of a body where magnitudes of both $\sigma_{z z}^{(n)}$ and $\sigma_{x z}^{(n)}$ exceed respective critical values. Such areas are considered to be the most damaged.

The analysis for the other moments of time and components of stress tensor is carried out similarly. 

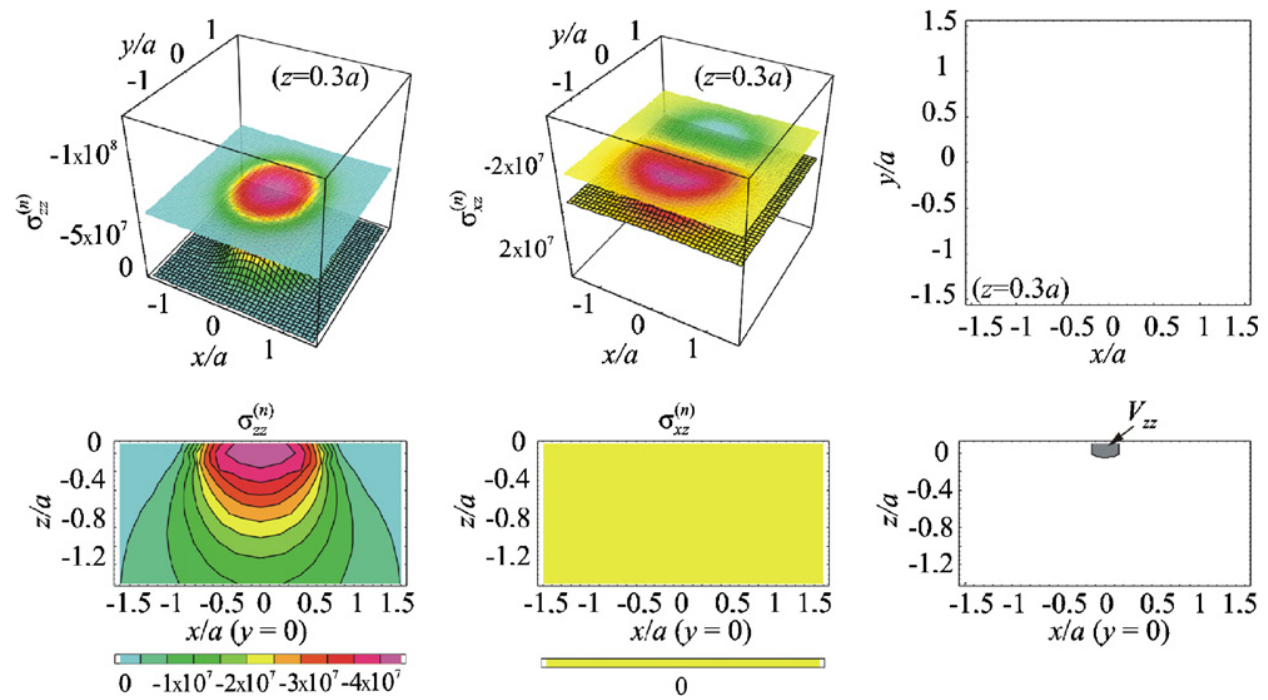

Fig. 13. Distribution of stresses $\sigma_{z z}$ and $\sigma_{x z}$ and respective dangerous volumes in planes $z=0.3$ a (top row) and $y=0$ (bottom row) at moment of time $t=4$.
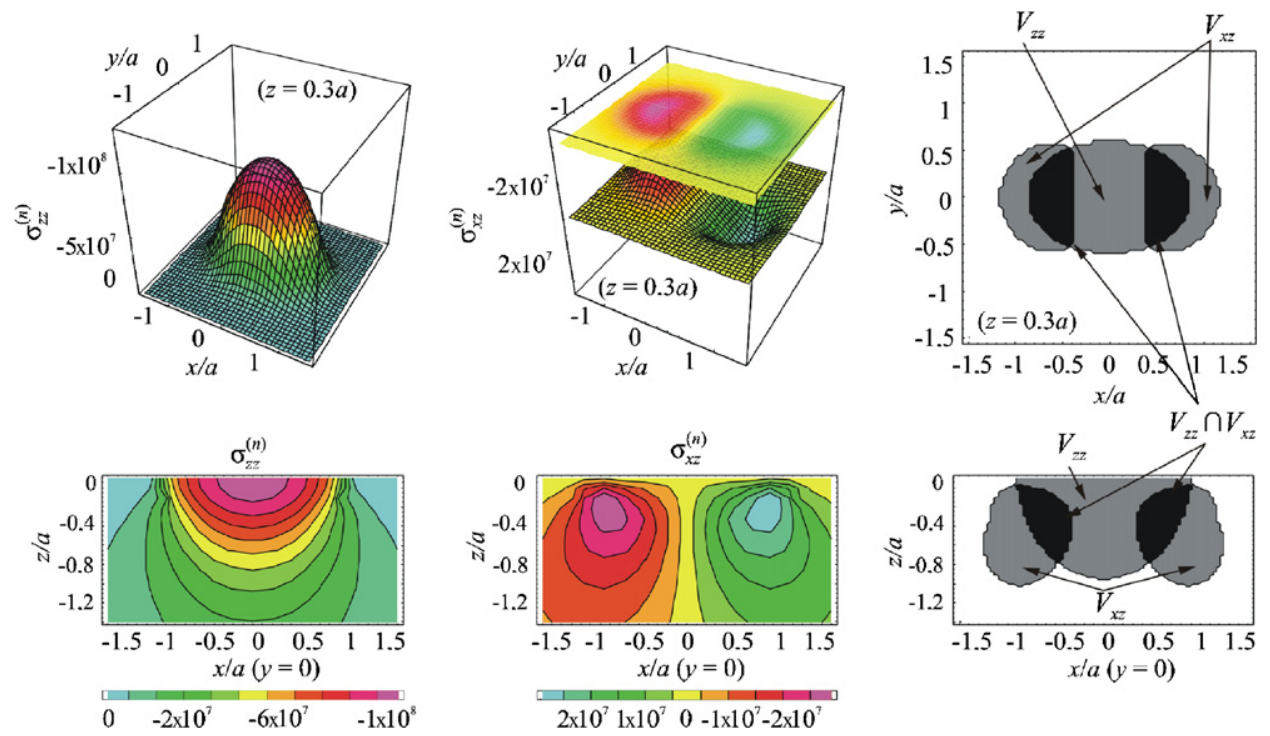

Fig. 14. Distribution of stresses $\sigma_{z z}$ and $\sigma_{x z}$ and respective dangerous volumes in planes $z=0.3$ a (top row) and $y=0$ (bottom row) at moment of time $t=6$.

Contact dynamics described theoretically is qualitatively confirmed by the experimental results [11]. The change of contact parameters in time determines the dynamics of formation of wavy damages in the conditions of rolling fatigue. However, it should be noted that there is no plasticity function in the theoretical solution. Nevertheless, elastoplastic behavior is considered implicitly by non-elastic change of geometric characteristics of the contact region. This change is also taken into account in the accepted model of a deformable rigid body with dangerous volume.

The scheme of analysis of the developed model is shown in Fig. 15. It shows a time interval. During this time interval the changes of main system parameters, stress state, and damage are sequentially calculated. These changes become the initial data for the calculation at the next time interval. 


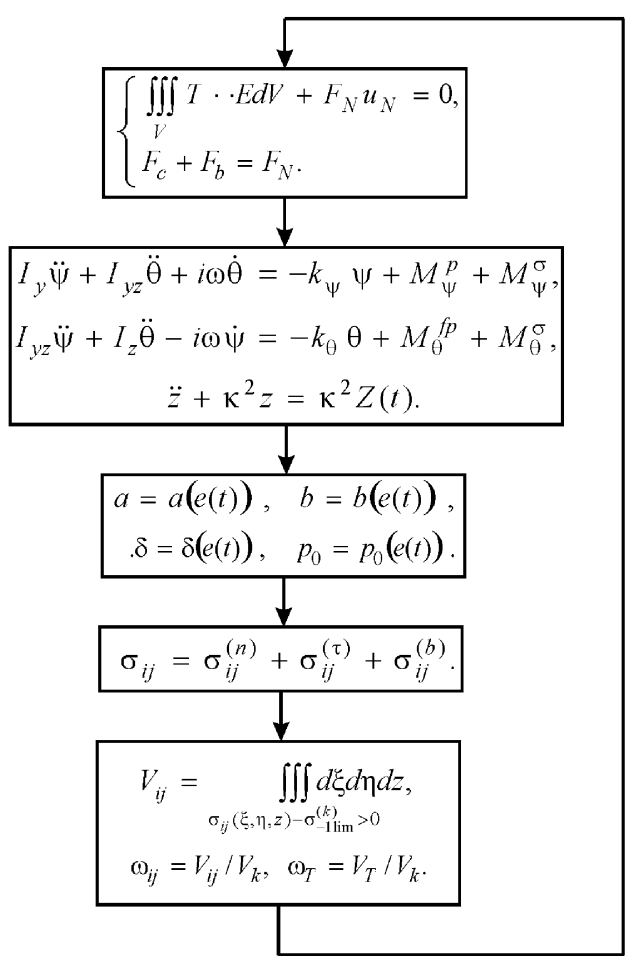

Fig. 15. Calculation procedure of a time interval for active system working in conditions of troppy.

\section{Main conclusions}

1. The results of systematic experimental research of troppy are presented.

2. The general calculation procedure for an active system working in the conditions of troppy is developed.

3. According to the statement of the problem, general differential equations (18) of the roller's movement on the shaft are built with regard to

- the roller's ability to oscillate in three planes;

- the characteristic features of geometry of interacting elements at the contact interface;

- heterogeneity of surface properties of material in local zones along the rolling track;

- disturbing action of external (contact and non-contact) loads.

It is shown that the non-stationary process of deformation in the analyzed conditions is accompanied by the fluctuations of all the parameters of contact and contact pressure as well.

4. Expressions (16) and (17) for the moments causing disturbance of the roller's movement are obtained analytically from the analysis of conditions of loading and contact interaction. Thus, the offered technique of modeling the disturbing action of loading factors is based on the determination of the integral moments by the distributed tractions transmitted to a roller on the surface of contact.

5. The problem of dynamic change of the state of stress in the neighborhood of contact is solved. Vibroimpact emerges as the result of drastic quasi-periodic change of contact pressure $p_{0}(x, y, t)$ accompanied by pulsations of displacement $\delta(t)$.

In our opinion, troppy can be considered as a fundamental phenomenon. In practice, its specific manifestation is, for example, rail corrugation. In certain conditions, troppy can lead to the occurrence of vibro-impact in the system. 


\section{References}

[1] L.A. Sosnovskiy, Tribo-Fatigue. Wear-Fatigue Damage and Its Prediction (Foundations of Engineering Mechanics), Springer, Berlin, 2005 424pp.

[2] L.A. Sosnovskiy, Fundamentals of Tribo-Fatuge, Vol. 2, Gomel, 2003, 246pp. (in Russian).

[3] GOST 30754-2001. Tribo-Fatigue. Methods of Wear-fatigue Tests. Tests for Mechano-Rolling Fatigue, Interstate Council on Standardization, Metrology and Certification, Minsk, 2002, 32pp. (in Russian).

[4] L.A. Sosnovskiy, A.V. Bogdanovich, S.A. Tyurin, Experiment Study of Mechano-Rolling Fatigue of Steel 45 by Multistep Loading, Zavodskaya Laboratoriya, Vol. 3, 1996, pp. 30-34 (in Russian).

[5] L.A. Sosnovskiy, S.S. Sherbakov, Troppy Phenomenon, Proceedings of World Tribology Congress III, 12-16 September 2005, Washington, DC, USA, 2pp.

[6] S.P. Timoshenko, J.N. Goodier, Theory of Elasticity, Nauka, Moscow, 1975, 576pp. (in Russian).

[7] V.I. Babitsky, Theory of Vibro-Impact Systems and Applications. (Foundations of Engineering Mechanics), Springer, 1998 306pp.

[8] M.V. Keldysh, Shimmy Motion of a Wheel of a Three Wheel Chassis. Selected Works, Mechanics, Moscow, 1985, pp. 491-530 (in Russian).

[9] I.G. Malkin, Theory of Dynamic Stability, Nauka, Moscow, 1966 530pp. (in Russian).

[10] K.L. Johnson, Contact Mechanics, Cambridge University Press, Cambridge, 1985 453pp.

[11] L.A. Sosnovskiy: Mechanics of Wear-Fatigue Damage, Gomel, 2006, 420pp. (in Russian). 